\title{
Kohlenoxydverbindungen der Nichtmetalle I
}

\author{
Darstellung und Eigenschaften von Carbonylselenid \\ Von Oskar Glemser und Teut Risler ${ }^{1}$ \\ Aus dem Institut für anorganische Chemie und Elektrochemie der Rheinisch-Westfäli- \\ schen Technischen Hochschule Aachen \\ (Z. Naturforschg. 3 b, 1-6 [1948]; eingegangen am 3. März 1947)
}

Leitet man Kohlenoxyd bei $780^{\circ} 2$ über Selen, dann entsteht Carbonylselenid. Die Ausbeute steigt mit erhöhter Strömungsgeschwindigkeit des Kohlenoxyds. Eine neue Darstellungsweise nach

$$
\mathrm{Al}_{2} \mathrm{Se}_{3}+3 \mathrm{COCl}_{2} \rightarrow 2 \mathrm{AlCl}_{3}+3 \mathrm{COSe}
$$

wird beschrieben und die Abhängigkeit der Ausbeute von der Reaktionstemperatur studiert. Bei $219^{\circ}$ und konstantem Durchsatz von $1,75 l \mathrm{COCl}_{2} /$ Stde. wird ein Ausbeutemaximum von $36 \%$ COSe gefunden. Das bei der Reaktion entstehende Aluminiumchlorid wirkt autokatalytisch auf den Ablauf der Reaktion ein.

Die Dampfdrucke des COSe werden durch die Tensionsgleichung log $p_{\mathrm{mm}}=$ $-1149,8 / T+7,4527$ wiedergegeben. Sdp. $-21,7^{\circ}$; Schmp. $-124,4^{\circ}$; mittlere molare Verdampfungswärme: $5260 \mathrm{cal}$; Troutonsche Konstante: 20,9 ; Litergewicht bei $0,0^{\circ}$ : $4,8272 \mathrm{~g}$; Dielektrizitätskonstante $\varepsilon_{0^{\circ}}=3,52$ (COSe flüss.); Dipolmoment $\mu=0,59 \cdot 10-18$ elektrostat. Einh. Die Löslichkeit in Phosgen bei $-44,0$ und $-32,5^{\circ}$ wird durch Dampfdruckmessungen verfolgt und eine Methode zur dielektrischen Bestimmung des COSeGehalts in Phosgen angegeben.

Das Verhalten des COSe gegenüber Säuren und Basen sowie oxydierenden Substanzen wird ermittelt. An porösen Körpern zerfällt COSe gegebenenfalls vollständig in Kohlenoxyd und Selen. Ganz trockenes COSe ist längere Zeit haltbar; in Gegenwart von Feuchtigkeit und Licht ist es wenig beständig, der Zerfall kann aber durch Phosgenzusatz gehemmt werden.

\footnotetext{
广 - ber Versuche zur Darstellung von Carbonylselenid berichtet erstmalig B e s s o ${ }^{3}$. Während er aus Schwefelwasserstoff in flüssigem Phosgen bei $220^{\circ}$ im Einschlußrohr Carbonylsulfid erhielt, gelang ihm die analoge Reaktion mit Selenwasserstoff nicht. v. B a t a l ${ }^{4}$ leitete gasförmiges Phosgen bei Rotglut über Cadmiumselenid; es entstand ein selenhaltiges Gas, das er bei $-80^{\circ}$ nicht verflüssigen konnte. Auch Berthelot ${ }^{5}$ konnte beim Erhitzen von Selen mit Kohlenoxyd im Rohr ein selenhaltiges Gas nachweisen. $\mathrm{P}$ e ar s on und $\mathrm{R}$ ob in s o ${ }^{6}{ }^{6}$ änderten die Methode von Berthelot ab, indem sie Kohlenoxyd über erhitztes Selen strömen ließen, wobei die Reaktion nach $\mathrm{CO}+\mathrm{Se} \rightarrow \mathrm{COSe}$ mit einer Ausbeute von etwa $4 \%$ eintrat. Sie charakterisierten Carbonylselenid als ein. farbloses, übelriechendes Gas, das bei $22,9^{\circ} / 725 \mathrm{~mm}$ siedet und bei $-122,1^{\circ}$ 1944.

1 Diss. Teut R i s l e r, Techn. Hochschule Aachen

${ }^{2}$ Alle Temperaturangaben in Celsiusgraden.

3 A. B e s s o n, C. R. hebd. Séances Acad. Sci. 122, 140 [1896].

4 A. v. B a r t a l, Chemiker-Ztg. 30, 1044 [1906].
}

zu schneeweißen Kristallen erstarrt. P u r ce 11 und $\mathrm{Z}$ a hoo $\mathrm{rbux}^{7}$ bestätigten diese Angaben; die von Pearson und Robinson angegebenen Dampfdruckwerte wurden von ihnen korrigiert. Sie fanden außerdem, daß die Ausbeute an Carbonylselenid durch Verwendung von Quarz- statt Glas- oder Porzellangefäßen erhöht wird. Weiterhin studierten sie den katalytischen Einfluß der verschiedenen Selenmodifikationen auf den Zerfall des Carbonylselenids bei 120 bis $140^{\circ}$ und stellten fest, daß die Zerfallsgeschwindigkeit durch $\beta$-Selen am stärksten beeinflußt wird.

Wir bedienten uns zuerst der Vorschrift von $\mathrm{P}$ earson und $\mathrm{R}$ obins on ${ }^{\mathbf{6}}$, um reines Carbonylselenid darzustellen, haben aber dann eine neue Synthese mit wesentlich besserer Ausbeute gefunden.

5 M. B erthel ot, Ann. chim. physique 22, 303 [1901].

6 T. G. P e a r s o n u. P. L. R ob in s on, J. chem. Soc. [London] 1932, 652.

7 R. H. Purcellu. F. D. Z a hoorbux, J. chem. Soc. [London] 1937, 1029. 


\section{Darstellung des Carbonylselenids}

a) Aus Kohlenoxydund Selen

Das aus 100-proz. Ameisensäure und konz. Schwefelsäure bei etwa $80^{\circ}$ entwickelte Kohlenoxyd wird durch 50-proz. Kalilauge geleitet, mit Ätzkali, Calciumchlorid und Phosphorpentoxyd getrocknet und durch einen Strömungsmesser (Rota) über Selen geführt, das in einem Quarzkolben auf $780^{\circ}$ erhitzt wird. Anschließend passiert das Gas verschiedene Kondensationsgefäße, die mit Eis, fester Kohlensäure und flüssiger Luft gekühlt werden. Das mit flüssiger Luft erhaltene Kondensat wird in einer Hochvakuumapparatur nach Stock fraktioniert; an Hand der Dampfdrucke wird die Reinheit festgestellt.

Zur Bestimmung der Ausbeute an Carbonylselenid wird das Gasgemisch durch eine Glasbirne von bekanntem Volumen geleitet. Die Zersetzung mit 2-n. Natronlauge nach

$$
\mathrm{COSe}+4 \mathrm{NaOH} \rightarrow \mathrm{Na}_{2} \mathrm{Se}+\mathrm{Na}_{2} \mathrm{CO}_{3}+2 \mathrm{H}_{2} \mathrm{O}
$$

ist nach etwa 15 Min. beendet. Danach gibt man die Lösung in ein Becherglas, leitet Luft ein ${ }^{8}$ und erhitzt, worauf sich schwarzes, elementares Selen abscheidet. Nach dem Abkühlen wird filtriert, mit Wasser und anschließend mit Methylalkohol gewaschen, bei $105^{\circ}$ getrocknet und das Selen gewogen.

In Ubereinstimmung mit $\mathrm{Purcel}$ und $\mathrm{Z}$ a h o o r $\mathrm{bux}^{7}$ beobachteten wir, daß bei Verwendung von Quarz- anstatt Glasgefäßen die Ausbeute etwas gesteigert wird. Eine weitere Steigerung der Ausbeute erzielten wir durch Erhöhung der Strömungsgeschwindigkeit des Kohlenmonoxyds (Tab.1).

\begin{tabular}{|c|c|c|}
\hline \multirow{2}{*}{$\underset{l / \text { Stde. }}{\text { Strömungsgeschw. }}$} & \multicolumn{2}{|c|}{$\begin{array}{c}\text { Ausbeute an COSe in Vol.. } \% \text { bei } \\
\text { Verwendung von }\end{array}$} \\
\hline & Supremaxglas & Quarzglas \\
\hline $\begin{array}{r}5 \\
15\end{array}$ & $\begin{array}{l}6,42 \\
9,05\end{array}$ & $\begin{array}{l}6,83 \\
9,84\end{array}$ \\
\hline
\end{tabular}

Tab. 1. Abhängigkeit der Ausbeute an COSe vom Material des Reaktionsgefäßes und der Strömungsgeschwindigkeit.

\section{b) Aus Aluminiumselenid und Phosgen}

Da die Ausbeute bei der Darstellung aus Kohlenoxyd und Selen unbefriedigend ist, versuchten wir, das Carbonylselenid durch Utberleiten von gasförmigem Phosgen über erhitztes Aluminiumselenid nach

$$
\mathrm{Al}_{2} \mathrm{Se}_{3}+3 \mathrm{COCl}_{2} \rightarrow 2 \mathrm{AlCl}_{3}+3 \mathrm{COSe}
$$

\section{zu gewinnen.}

8 Diese Methode ist nach unseren Erfahrungen nur bei kleinen Mengen Selen geeignet.

$\theta$ H. G. Grim m u. H. Metzger, Ber. dtsch. chem. Ges. 69, 1356 [1936].
Darstellung von Aluminiumselenid:

Aluminiumselenid wird durch Zünden eines Gemisches von $1 \mathrm{Tl}$. Aluminium mit 7 Tln. rotem Selen mit Magnesiumband dargestellt ${ }^{9}$. Nach unseren Erfahrungen ist es zweckmäßig, das Gemisch vor der Zündung über Nacht über Phosphorpentoxyd stehen zu lassen, da sonst nicht ein fester Kuchen von Aluminiumselenid, sondern ein grießiges Produkt ${ }^{10}$ entsteht. Besser stellt man Aluminiumselenid her, wenn man stöchiometrische Mengen von Aluminium und rotem Selen gut vermengt und die Mischung in ein evakuiertes Bombenrohr einschmilzt. Durch vorsichtiges Erhitzen mit freier Flamme kommt bei beginnender Rotglut die Reaktion mit heller Lichterscheinung in Gang und schreitet langsam durch die ganze Masse fort. Das erhaltene Produkt entwickelt mit Salzsäure stürmisch Selenwasserstoff und ist nach Debye-Scherrer-Aufnahmen identisch mit dem nach dem ersten Verfahren gewonnenen.

Reinigung von Phosgen: Das der Bombe entnommene Phosgen wird durch konz. Schwefelsäure und über Phosphorpentoxyd geleitet, in einem Eisbad kondensiert und im Hochvakuum bis zur Konstanz der Dampfdrucke fraktioniert.

Apparatur und Versuchsmethodik:

In einem Röhrenofen, der durch einen Spannungsregler auf konstanter Temperatur gehalten wird, befindet sich ein Rohr aus schwer schmelzbarem Glas, dessen eine Seite mit einem Gefäß für das Phosgen verbunden ist, die andere Seite ist über zwei Kondensationsgefäße an die Hochvakuumapparatur angeschlossen. Das Reaktionsrohr wird mit zerkleinertem Aluminiumselenid gefüllt und 12 Stdn. bei $350^{\circ}$ im Hochvakuum ausgeheizt ${ }^{11}$. Dann wiegt man gereinigtes Phosgen in ein Wägegefäß nach $S$ to $\mathrm{ck}^{\mathbf{1 2}}$ ein und destilliert es in das oben beschriebene Gefäß. Dieses wird durch ein Kältebad auf eine bestimmte Temperatur gebracht, die Kondensationsgefäße werden mit flüssiger Luft gekühlt und die Verbindung aller Gefäße zum evakuierten Reaktionsrohr hergestellt. Das Phosgen streicht über das erhitzte Aluminiumselenid, die Reaktionsprodukte und unverbrauchtes Phosgen werden im ersten Kondensationsgefä $\beta$ ausgefroren. Je nach Temperatur. des Kältebads stellt sich der Dampfdruck des Phosgens ein; je geringer der Dampfdruck, um so länger dauert der Durchsatz des Phosgens, d. h. um so kleiner ist die Strömungsgeschwindigkeit. Zur Bestimmung der Strömungsgeschwindigkeit wird in einem Glaskolben von bekanntem Volumen, der nach dem Reaktionsrohr angebracht ist, bei nicht erhitztem Aluminiumselenid das in 1 Stde. übergegangene Phosgen ausgefroren, hernach verdampfen gelassen und die Menge Phosgen

10 Selen darf aber vorher nicht für sich getrocknet werden, sonst geht die Reaktion nachher schlecht vonstatten.

${ }^{11}$ Bei der Durchführung der Reaktion muß das Vorhandensein von Wasser oder Wasserdampf peinlichst vermieden werden, da aus Aluminiumselenid sonst Selenwasserstoff frei wird.

12 A. S t o c k, Ber. dtsch. chem. Ges. 50, 156 [1917]. 
manometrisch gemessen. Ist im Phosgengefäß alle Substanz verdampft, dann ist der Versuch zu Ende. Mit einer Füllung von Aluminiumselenid können etwa 20 Versuche durchgeführt werden.

Zur Aufarbeitung der Reaktionsprodukte destilliert man alle bei Zimmertemperatur flüchtigen Bestandteile in das zweite Kondensationsgefäß. Als Rückstand hinterbleibt ein unbedeutender roter Beschlag von Selen. Im zweiten Kondensationsgefäß befindet sich unverbrauchtes Phosgen und Carbonylselenid, denn bei einer Badtemperatur von $-116^{\circ}$ (Alkoholbad) kann reines Carbonylselenid aus dem Gemisch im Hochvakuum abdestilliert werden, der Rest besteht nach Dampfdruckmessungen aus Phosgen.

Bei den Versuchsreihen wurde das im zweiten Kondensationsgefäß befindliche Gemisch von Phos-

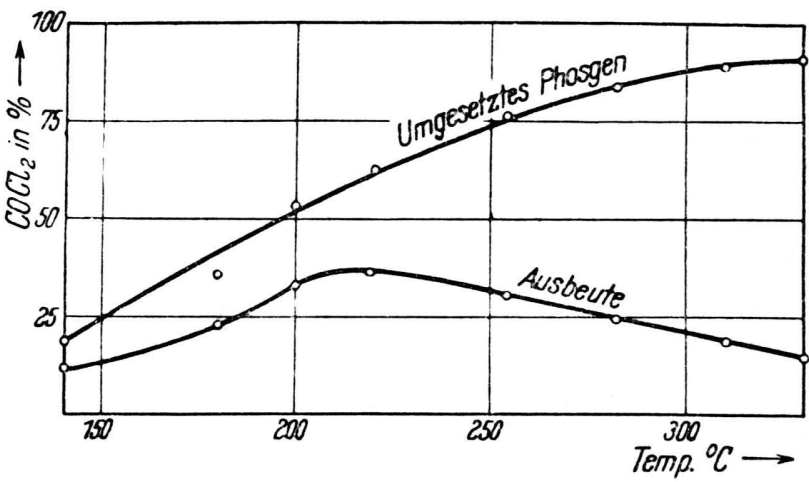

Abb. 1.

gen und Carbonylselenid zuerst gewogen (Destillat) und dann in einem Gasabsorptionsgerät, wie es in Orsat-Apparaten verwendet wird, mit Kaliumhypobromitlösung verseift und oxydiert. Zuerst scheidet sich etwas rotes Selen ab, das aber sofort zu Selenit oxydiert wird. Nach Beendigung der Reaktion wird die Lösung in einem Becherglas mit konz. Salzsäure bis zur schwach sauren Reaktion versetzt und bei $70^{\circ}$ mit Hydrazinsulfat zu elementarem Selen reduziert. Hierbei darf die selenhaltige Lösung nicht ins Sieden geraten, da sich das schwarze Selen sonst an der Flüssigkeitsoberfläche und der oberen Wand des Becherglases als dünne Haut abscheidet, die nur unter Substanzverlust wieder abgelöst werden kann. Das ausgefällte rote Selen wandelt sich in der Wärme schnell in die schwarze Modifikation um. Nach dem Abkühlen wird filtriert, mit Wasser und Methylalkohol gewaschen, bei $105^{\circ}$ getrocknet und gewogen.

Ausbeute: Der analytisch ermittelte COSe-Gehalt des Destillats wird als Prozentsatz der COSe-Menge in $g$ berechnet, die sich bei Annahme völligen Umsatzes aus dem eingewogenen Phosgen ergibt.

In Abb. 1 sind die Werte des umgesetzten Phosgens als Prozentsatz des eingesetzten Phosgens sowie die Ausbeute an COSe (umgerechnet auf $\mathrm{COCl}_{2}$ ) in Abhängigkeit von der Reaktionstemperatur eingetragen (in Tab. 2 sind einige Versuchs-
Beis piel:

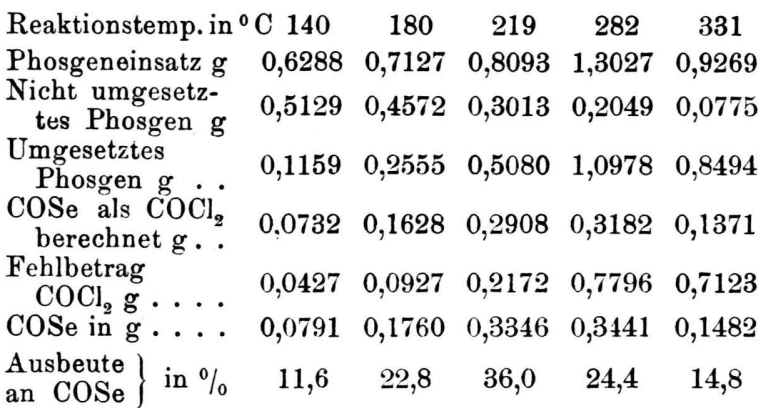

Tab. 2. Versuchsreihe V, Durchsatz $1,75 l \mathrm{COCl}_{2} /$ Stde.

daten der Versuchsreihe V angegeben). Die Ausbeute steigt mit höher werdender Temperatur bis $\mathrm{zu}$ einem Maximalwert von $36 \%$ bei $219^{\circ}$ an und sinkt wieder ab bei weiter steigenden Temperaturen; das umgesetzte Phosgen dagegen nimmt relativ gleichmäßig zu. Die Diskrepanz zwischen umgesetztem Phosgen und gebildetem Carbonylselenid ist in Tab. 2 unter Fehlbetrag $\mathrm{COCl}_{2}$ aufgenommen. In Abb. 1 ergibt der Unterschied der Werte des umgesetzten Phosgens von den Werten der Ausbeute den Fehlbetrag an $\mathrm{COCl}_{2}$ direkt in \%. Zur Erklärung kann man annehmen, daß das Phosgen einer thermischen Spaltung unterliegt, bzw. daß Nebenreaktionen eintreten etwa nach: $\mathrm{COSe}+2 \mathrm{Cl}_{2} \rightarrow \mathrm{CO}+\mathrm{SeCl}_{4} ; \mathrm{Se}+2 \mathrm{Cl}_{2} \rightarrow \mathrm{SeCl}_{4}$, oder daß das COSe seinerseits eine thermische Spaltung erleidet.

Beginnt man einen Versuch mit frischem Aluminiumselenid, so liegt die Ausbeute an Carbonylselenid bei $1-2 \%$ (Tab. 3). Der prozentuale Fehlbetrag an $\mathrm{COCl}_{2}$, bezogen auf das umgesetzte Phosgen, ist dann am höchsten und sinkt bei weiteren Versuchen auf einen konstanten Wert, während die Ausbeute steigt. Es wird zunächst, wie weiter unten noch dargelegt wird, Aluminiumchlorid gebildet, auch entsteht $\mathrm{SeCl}_{4}$, das wir als Sublimationsprodukt am Ende des Rohres nachgewiesen haben. Ist genügend katalytisch wirksames (s. weiter unten) Aluminiumchlorid vorhanden, entsteht mehr Carbonylselenid, und der Fehlbetrag an $\mathrm{COCl}_{2}$ verringert sich. Je mehr Versuche mit der gleichen Rohrfüllung durchgeführt werden, um so mehr ist am Ende des Reaktionsrohres ein roter Beschlag von Selen wahrzunehmen, der von zersetztem Carbonylselenid herrühren kann. Da Selen den Zerfall von COSe in $\mathrm{CO}$ und Se katalysiert ${ }^{7}$, ist bei der 
Reaktion auch mit einer Spaltung des Carbonylselenids zu rechnen. Der Prozentsatz nicht umgesetzten Phosgens nimmt nun mit zunehmender Ausbeute an COSe stark ab (Tab.3); der Fehlbetrag an $\mathrm{COCl}_{2}$ ist also hauptsächlich durch die Zersetzung des Carbonylselenids bedingt. Temperaturerhöhung bei der Reaktion ergibt stärkeren Zerfall des Carbonylselenids, aber auch des Phosgens ${ }^{13}$, wodurch immer größere Fehlbeträge an $\mathrm{COCl}_{2}$ sich ergeben müssen, wie auch aus Abb. 1 hervorgeht. Das vom Zerfall des Phosgens stammende Chlor setzt sich weiter zu Aluminiumchlorid bzw. $\mathrm{SeCl}_{4}$ um; elementares Chlor haben wir nie in den Reaktionsprodukten gefunden.

\begin{tabular}{|c|c|c|c|c|c|}
\hline Versuchsnummer .... & 1 & 2 & 3 & 4 & 5 \\
\hline $\begin{array}{l}\text { Nicht umgesetztes Phos- } \\
\text { gen in }\end{array}$ & 90,5 & 57,3 & 30,2 & 26,0 & 26,0 \\
\hline $\begin{array}{l}\text { Umgesetztes Phosgen in } \% \\
\text { Fehlbetrag Phosgen in } \%\end{array}$ & 9,5 & 42,7 & 69,8 & 74,0 & 74,0 \\
\hline $\begin{array}{l}\text { bezogen auf umgesetz- } \\
\text { tes Phosgen........ }\end{array}$ & 82,1 & 58,5 & 64,0 & 65,2 & 64,6 \\
\hline Ausbeute an COSe i & 1,7 & 17,8 & $2 \check{5}, 1$ & 25,7 & 26,0 \\
\hline
\end{tabular}

Tab. 3. Versuchsreihe II, Reaktionstemperatur $330^{\circ}$, Durchsatz $1,18 l \mathrm{COCl}_{2} /$ Stde.

Auffällig ist die Erscheinung, daß bei jeder neuen Beschickung des Rohres mit Aluminiumselenid die Ausbeute an Carbonylselenid zunächst bei 1-2\% liegt (vgl. Tab. 3) und schon nach wenigen Versuchen auf die der Reaktionstemperatur und dem Durchsatz entsprechende Höhe steigt. Mischt man aber dem Aluminiumselenid von vornherein Aluminiumchlorid zu, dann entspricht die Ausbeute schon beim ersten Durchsatz der erwarteten Höhe. Damit ist die katalytische Wirkung des Aluminiumchlorids bei der vorliegenden Reaktion $\mathrm{Al}_{2} \mathrm{Se}_{3}+3 \mathrm{COCl}_{2} \rightarrow 2 \mathrm{AlCl}_{3}+$ 3 COSe erwiesen; der Katalysator bildet sich von selbst bei der Reaktion: es liegt Autokatalyse vor.

Läßt man die Reaktion bei Normaldruck ablaufen, dann ist die maximale Ausbeute an COSe etwa $12 \%$. Nimmt man Aluminiumchlorid als sich im Dampfzustand befindend an, dann ist die Zahl der gasförmigen Molekeln auf der rechten Seite der Reaktionsgleichung $\mathrm{Al}_{2} \mathrm{Se}_{3}+3 \mathrm{COCl}_{2} \rightarrow 2 \mathrm{AlCl}_{3}$ +3 COSe größer als auf der linken: die Ausbeute liegt bei niederem Druck höher.

\footnotetext{
13 M. Bodenstein u. H. Plaut, Z. physik. Chem. 110, 399 [1924].

14 Bei der Herstellung der Dampfdruckthermometer und bei der Bestimmung der Löslichkeit von COSe in $\mathrm{COCl}_{2}$ unterstützte uns Hr. Dr. S a u e r.
}

\section{Eigensehaften des Carbonylselenids}

a) Physikalische Eigenschaften

Das für die Bestimmung der physikalischen Eigenschaften verwendete Carbonylselenid wurde durch fraktionierte Destillation und Kondensation im Hochvakuum bis zur Konstanz der Dampfdrucke gereinigt. Aus verschiedenen Versuchen nach beiden Darstellungsmethoden sind nachfolgende Dampfdruckwerte erhalten worden, die sich von den Werten von $\mathrm{Purcell}$ und $\mathrm{Z}$ ahoor$\mathrm{bux}^{7}$ etwas unterscheiden (Tab.4).

Dampidrucke $^{14}$. Zur Bestimmung der Dampfdrucke dienten als Kühlbäder Schmelzen von Tetrachlorkohlenstoff, von Monochlorbenzol, Monobrombenzol, Isobuttersäure und ein Gemisch einer Schmelze von Monochlor- und Monobrombenzol. Die Temperatur wurde mit Dampfdruckthermometern nach $\mathrm{St}$ o $\mathrm{ck}^{15}$ ermittelt, der Meniskus mit einem Kathetometer auf $0,1 \mathrm{~mm}$ genau abgelesen.

$\begin{array}{lrrrrc}\mathrm{Temp}^{\circ}{ }^{\circ} \mathrm{C} & -22,9_{1} & -31,3_{7} & -36,7_{7} & -44,3_{4} & -52,6_{\mathrm{o}} \\ p_{\mathrm{mm} \mathrm{Hg}} & 720,9 & 498,7 & 387,5 & 267,7 & 172,7 \\ \begin{array}{l}\text { beobachtet } \\ p_{\text {mm Hg }}\end{array} & 721,3 & 498,0 & 387,7 & 267,7 & 173,5 \\ \text { berechnet } & & & & & \end{array}$

Tab. 4. Dampfdruckwerte von COSe.

Die Dampfdruckwerte werden durch die Tensionsgleichung

$$
\log p=-1149,8 / T+7,4527
$$

wiedergegeben. Daraus errechnet sich der Siedepunkt $\mathrm{zu}-21,7^{\circ}$; die mittlere molare Verdampfungswärme ist 5260 cal. Die Troutonsche Konstante ergibt sich zu 20,9.

Schmelzpunkt nach $\mathrm{S}$ to $\mathrm{ck}^{12}$ im Hochvakuum bestimmt, Mittelwert aus fünf Bestimmungen: $-124,37 \pm 0,05^{\circ}$.

Bei $760 \mathrm{~mm} \mathrm{Hg}$ und $0,0^{\circ}$ wiegt $1 l$ COSe $4,8272 \mathrm{~g}$ (Mittelwert aus 4 Best.). Danach ist das Molekulargewicht von COSe 108,3, ber. 106,97. COSe liegt also bei $0,0^{\circ}$ in monomolekularer Form vor.

Die Dielektrizitätskonstante wurde mit Hilfe des nach der Überlagerungsmethode arbeitenden Dielkometers ${ }^{16}$ gemessen. In Anlehnung an eine frühere Konstruktion ${ }^{17}$ bauten wir den in Abb. 2

15 A. S t o c k, Ber. dtsch. chem. Ges. 54, 1119 [1921];

Z. Elektrochem. angew. physik. Chem. 29, 355 [1923].

16 Hersteller: $\mathrm{H}$ a a r d t u. Ci e. AG., Düsseldorf.

17 O. G l e m s e r, Z. Elektrochem. angew. physik. Chem. 44, 341 [1938]. 
gezeichneten Kondensator, der den Anschluß an die Hochvakuumapparatur erlaubte. Die Eichung des Kondensators erfolgte mit Dioxan-Wasserge-

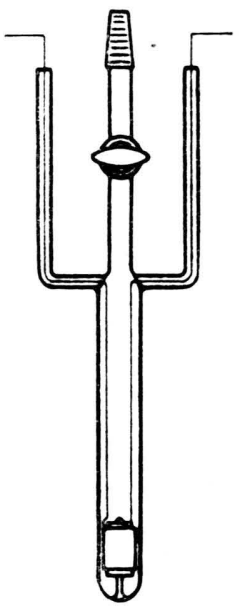

Abb. 2

Kondensator zur Messung der DK von COSe. mischen bekannter Dielektrizitätskonstante (DK) und mit im Hochvakuum gereinigtem Benzol. Bei den Messungen blieb der Hahn zum Hochvakuum geschlossen.

Tab. 5 verzeichnet die DKWerte von COSe bei vier verschiedenen Temperaturen (COSe in flüssigem Zustand).

$$
\begin{array}{ccccc}
t_{0} & -54,0 & -7,6 & 0,0 & 10,0 \\
\mathrm{DK} & 3,79 & 3,56 & 3,53 & 3,47
\end{array}
$$

Tab. 5. DK von COSe in Abhängigkeit von der Temperatur.

Aus den DK-Werten errechnet sich - unter der vereinfachenden Annahme, daß die Gesetzmäßigkeit für den Dampf auch für die Flüssigkeit bei hohem Dampfdruck gültig sei - das Dipolmoment $\mu=0,59 \cdot 10^{-18}$ elektrostat. Einheiten ${ }^{18}$.

Die Bestimmung der DK kann auch zu einer schnellen Ermittlung des Gehalts an COSe in $\mathrm{COCl}_{2}$ benützt werden. In Tab. 6 sind einige Meßwerte eingewogener Gemische von COSe und $\mathrm{COCl}_{2}$ eingetragen. Die Werte liegen, graphisch dargestellt, auf einer Geraden.

$\begin{array}{lccccc}\text { Konz.an COSe in } \% & 100,0 & 65,6 & 40,8 & 30,7 & 0,0 \\ \text { Feinkondensator . . } & 73,2 & 68,4 & 65,5 & 64,4 & 60,9 \\ \text { DK . . . . . . } & 3,53 & 3,99 & 4,27 & 4,38 & 4,79^{19}\end{array}$

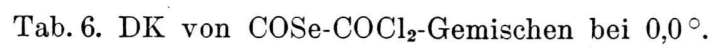

Löslichkeit von COSe in $\mathrm{COCl}_{2}$ : Als Kriterium für die Löslichkeit des Carbonylselenids in Phosgen dienen die in der Hochvakuumapparatur gemessenen Dampfdrucke von Gemischen bekannter Zusammensetzung. Die Gemische werden hergestellt, indem in einen Glaskolben bekannten Inhalts jeweils von beiden Substanzen eine bestimmte Menge, gemessen an dem im Kolben herrschenden Druck, eingelassen wird. Das Gas wird dann wieder ausgefroren und in das Gefäß destilliert, in dem die Löslichkeitsbestimmung

18 A. Eucken, Grundr. d. physik. Chem., S. 517, Leipzig 1942.

${ }^{19} 4,79$ ist die DK von flüssigem Phosgen bei $0,0^{\circ}$. vorgenommen wird. Die Dampfdrucke der Gemische werden bei $-44,0$ und $-32,5^{\circ}$ gemessen (vgl. Tab. 7A, 7B u. Abb. 3).

$$
\begin{aligned}
& \text { A. Temp. }-44,0^{\circ}
\end{aligned}
$$

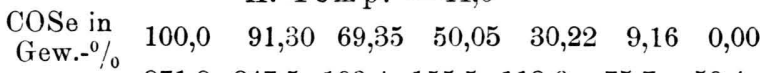

$$
\begin{aligned}
& \begin{array}{llllllll}
p_{\mathrm{mm} \mathrm{Hg}} & 271,8 & 247,5 & 193,4 & 155,5 & 118,6 & 75,7 & 59,4
\end{array} \\
& \text { B. Tem p. }-32,5^{\circ}
\end{aligned}
$$

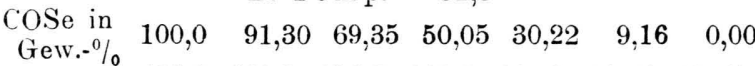

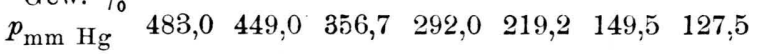

Die Kurvenzüge verlaufen in Abb. 3 kontinuierlich, Kohäsionskräfte sind zwischen COSe und $\mathrm{COCl}_{2}$ bei diesen Temperaturen nicht anzunehmen.

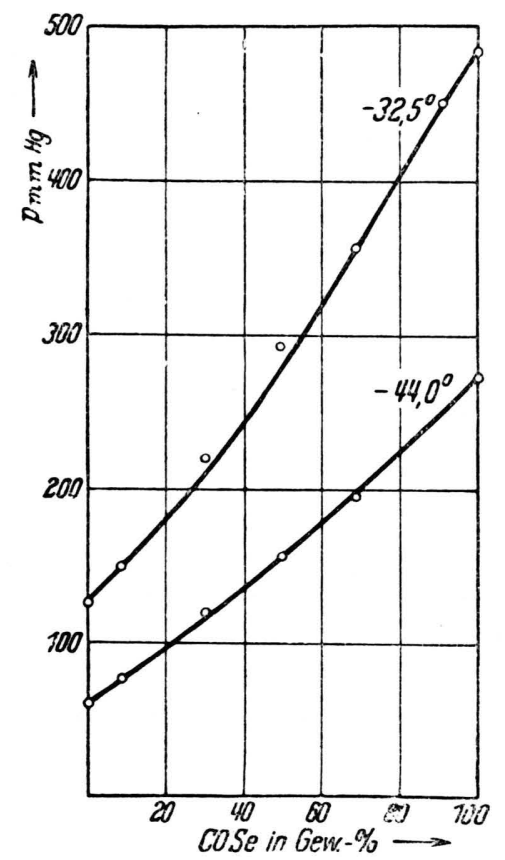

Abb. 3. Löslichkeit von COSe in $\mathrm{COCl}_{2}$ bei $-44,0$ und $-32,5^{\circ}$.

b) Chemische Eigenschaften

Zur Untersuchung kamen die flüssigen Stoffe in eine Waschflasche, durch die Carbonylselenid mit mäßiger Geschwindigkeit geleitet wurde. Feste Reagenzien kamen in ein mit Normalschliffen versehenes Glasrohr, das an das Vorratsgefäß für COSe angeschlossen wurde. Als Trägergas diente trockener Stickstoff, wenn COSe nicht in einem Quecksilbergasometer aufbewahrt wurde. Bei allen Versuchen wurde das die Apparatur 
verlassende Gas auf unzersetztes COSe untersucht. Zur qualitativen Prüfung auf Kohlenmonoxyd wurde $\mathrm{PdCl}_{2}$-Lösung vorgelegt, die gegen die Einwirkung von Wasserstoff mit Natriumsulfit und Natriumacetat geschützt war.

Konz. Schwefelsäure färbt sich schwach gelb. Beim Verdünnen mit Wasser fällt rotes Selen aus. Die Absorption von COSe ist gering. Konz. Salpetersäure oxydiert zum Selenit $\mathrm{SeO}_{3}{ }^{\prime \prime}$. Beim Reduzieren mit Schwefliger Säure ist danach eine schwache Selenabscheidung zu beobachten (nach Vertreiben der Stickoxyde). Perhydrol wirkt wie konz. Salpetersäure. Salzsäure hydrolysiert ebenfalls unter Abscheidung von Selen, doch geht die Reaktion sehr langsam und nur in geringem Maße vor sich. Dagegen hydrolysieren Alkalien schnell und quantitativ. Natronlauge, Kalilauge, Ammoniaklösung, Sodalösung reagieren vollständig nach

$$
\mathrm{COSe}+4 \mathrm{NaOH} \rightarrow \mathrm{Na}_{2} \mathrm{Se}+\mathrm{Na}_{2} \mathrm{CO}_{3}+2 \mathrm{H}_{2} \mathrm{O} .
$$

Die Lösung färbt sich bei wenig COSe gelb, bei mehr orangerot. Durch Oxydation des Selenids bilden sich Polyselenide, die aber nach einigem Aufbewahren an der Luft zerfallen und Selen abscheiden. Fügt man zu der gelben Lösung Wasserstoffperoxyd, dann entfärbt sie sich unter Bildung von $\mathrm{SeO}_{3}{ }^{\prime \prime}$.

Feste Alkalien, z.B. Natronkalk, färben sich durch abgeschiedenes Selen rot. Bimsstein, der mit Alkalicarbonat ${ }^{50}$ getränkt und bei $100^{\circ}$ getrocknet wird, gibt eine unvollständige Spaltung in Kohlenoxyd und Selen.

Quantitative Untersuchungen (Quecksilbergasometer, Orsat-App.) an einigen porösen Körpern, z.B. Aktivkohle, ergaben, daß Carbonylselenid allein oder in jedem Mischungsverhältnis mit Phosgen sich vollkommen in Kohlenmonoxyd und Selen spaltet. Tränkt man aber solche Körper mit alkal. Schwermetallsalzlösungen, z. B. Silbernitrat in Ammoniak oder Triäthanolamin, so zersetzt sich Carbonylselenid an dem porösen Körper unter Bildung von $\mathrm{Ag}_{2} \mathrm{Se}$ und Alkalicarbonat.

Einwirkung von Wasser und Licht: Vollkommen trockenes Carbonylselenid ist im Dunkeln tagelang haltbar. Bei Belichtung zerfällt COSe,

\footnotetext{
20 Gehalt an $\mathrm{K}_{2} \mathrm{CO}_{3}$ beispielsweise $4 \%$.
}

was qualitativ durch die Selenabscheidung an der Gefäßwand des Kolbens zu beobachten ist.

In einen im Hochvakuum ausgeheizten Glaskolben von $650 \mathrm{~cm}^{3}$ Inhalt wird soviel reines, trockenes COSe eingewogen, daß der Druck 0,36 atm beträgt. Nach 20 Stdn. Belichtung (Tageslicht, Lufttemp. 25 ${ }^{\circ}$ ) hat sich etwas Selen an der Wand des Gefäßes abgeschieden. Nach Abpumpen des restlichen Gases wird das anhaftende Selen mit rauchender Salpetersäure gelöst, die Lösung zweimal mit konz. Salzsäure abgeraucht und aus der Lösung durch Einleiten von $\mathrm{SO}_{2}$ Selen gefällt.

Eingesetztes COSe: $1,0468 \mathrm{~g}$; abgeschiedenes Selen : 0,0143 g Zersetztes COSe: 0,0194g; entspr. 1,85\%.

Ist Wasser zugegen, dann zersetzen sich bei 20-stdg. Belichtung und einem Wasserdampfdruck von $15 \mathrm{~mm} \mathrm{Hg}$ im Kolben 12\% COSe. Wir fanden aber, daß Phosgen die Zersetzung von Carbonylselenid bei Gegenwart von Wasser bzw. Wasserdampf hemmt (s. Tab.8).

In einen Kolben werden bestimmte Mengen COSe und $\mathrm{COCl}_{2}$ eingewogen. Bei Zimmertemperatur werden sie im Kolben verdampft, der Rest des Kolbenvolumens wird so mit Luft gefüllt, die zuvor eine Waschflasche mit Wasser von $20^{\circ}$ passiert hat, daß hernach im Kolben ein geringer Unterdruck von 5-10 mm herrscht. Nach Beendigung des Versuchs wird das am Kolben abgeschiedene Selen, wie oben beschrieben, bestimmt.

$\begin{array}{lccc}\text { COSe : } \mathrm{COCl}_{2} \ldots \ldots & 1: 0,55 & 1: 1,93 & 1: 7,6 \\ \text { Kolbeninhalt in } \text { cm }^{3} & 650 & 650 & 5000 \\ \text { Zersetztes COSe in } \% & 3,24 & 1,51 & 1,31\end{array}$

Tab. 8. Einfluß von Licht und Wasserdampf auf Gemische von $\mathrm{COSe}$ und $\mathrm{COCl}_{2}$. Versuchsdauer 6 Tage: Lufttemp. $20-25^{\circ}$.

Es bleibt dahingestellt, ob Carbonylselenid primär durch Licht zerfällt oder Reste von Feuchtigkeit Hydrolyse auslösen, die Selenwasserstoff entstehen läßt, der unter Licht- und Lufteinwirkung zu Selen oxydiert wird. Am Selen kann dann weiterhin eine katalytische Zersetzung des Carbonylselenids stattfinden, wobei die Einwirkung von Licht ebenfalls eine Rolle spielen kann. Auch die Glasoberfläche kann eine katalytische Zersetzung des COSe begünstigen oder auch chemisch infolge seiner Alkalität wirken.

Hrn. Prof. Dr. A. B e n r a th danken wir für wohlwollende Unterstützung. Der Deutschen For $\mathrm{schungsgemeinschaft} \mathrm{sind} \mathrm{wir} \mathrm{für} \mathrm{Überlas-}$ sung wertvoller Apparate zu Dank verpflichtet. 